\title{
Predictors for the use of traditional Chinese medicine among inpatients with first-time stroke: a population-based study
}

Wei-Sen Chen ${ }^{1,2}$, Hung-Chih Hsu ${ }^{1,3,4,5,6,7}$, Yi-Wen Chuang ${ }^{1,8}$, Meng Lee ${ }^{3,9}$, Kuan-Yu Lu' ${ }^{1}$, Yi-Fei Chen ${ }^{10}$ and Chien-Min Chen ${ }^{1,3^{*}}$ (1)

\begin{abstract}
Background: Stroke is one of the major causes of death and disability. The treatments that are provided to patients during hospitalization after an acute stroke are very important in stabilizing their medical condition and enabling the recovery of their motor functions. However, limited information is available regarding the use of traditional Chinese medicine (TCM) during hospitalization for first-time stroke patients. The researchers aimed to investigate the factors affecting TCM use and to provide clinicians with comprehensive information on TCM use among first-time stroke inpatients in Taiwan.
\end{abstract}

Methods: The researchers collected and analyzed data, including patient characteristics, TCM use, and TCM prescription patterns, from the National Health Insurance Research Database in Taiwan for first-time stroke inpatients between 2006 and 2012.

Results: Among the 89,162 first-time stroke patients, 7455 were TCM users, and 81,707 were TCM nonusers. The predictors for TCM use were as follows: age, 45-64 or < 45 years; men; living in a level 2, 4, or 7 urbanized area; insured amount $\geq 576$ USD per month; ischemic stroke; hospitalized for first-time stroke for 8-14 days, 15-28 days, or $\geq 29$ days; stroke severity index score $0-9$ or 10-19; Charlson-Deyo comorbidity index score 0 or 1-2; hospitalization in a regional or community hospital; receiving rehabilitation; and previous experience with outpatient TCM use. An increase in the number of TCM users was observed from 2006 to 2012. Furthermore, 68.879.7\% of TCM users used acupuncture only, while 17.8-26.1\% used both acupuncture and Chinese herbal medicine.

Conclusions: An increasing number of first-time stroke patients have been choosing TCM as a complementary treatment during hospitalization. Moreover, TCM use is associated with demographic, clinical, and socioeconomic characteristics. These findings may help clinicians comprehensively understand the trend and the important factors affecting TCM utilization among patients who are hospitalized due to first-time stroke.

Keywords: Traditional Chinese medicine, Stroke, National Health Insurance Research Database, Complementary and alternative medicine

\footnotetext{
* Correspondence: fateman53@yahoo.com.tw

'Department of Physical Medicine and Rehabilitation, Chang Gung Memorial

Hospital, Chiayi, No.6, W. Sec., Jiapu Rd., Puzih City, Chiayi County 613,

Taiwan

${ }^{3}$ School of Medicine, College of Medicine, Chang Gung University, Taoyuan,

Taiwan

Full list of author information is available at the end of the article
}

(c) The Author(s). 2020 Open Access This article is licensed under a Creative Commons Attribution 4.0 International License, which permits use, sharing, adaptation, distribution and reproduction in any medium or format, as long as you give appropriate credit to the original author(s) and the source, provide a link to the Creative Commons licence, and indicate if changes were made. The images or other third party material in this article are included in the article's Creative Commons licence, unless indicated otherwise in a credit line to the material. If material is not included in the article's Creative Commons licence and your intended use is not permitted by statutory regulation or exceeds the permitted use, you will need to obtain permission directly from the copyright holder. To view a copy of this licence, visit http://creativecommons.org/licenses/by/4.0/. The Creative Commons Public Domain Dedication waiver (http://creativecommons.org/publicdomain/zero/1.0/) applies to the data made available in this article, unless otherwise stated in a credit line to the data. 


\section{Background}

Stroke is one of the major causes of death worldwide [1]. Poststroke complications and functional impairment may lead to long-term disability [2]. In Taiwan, the incidence and prevalence rates of stroke are 330/100,000 person-years and 19.3/1000, respectively [3,4], and the national expenditure for stroke is more than 400 million United States dollar (USD) per year [5], thus making it a large healthcare burden.

Moreover, $95 \%$ of stroke survivors show significant functional recovery within 3 months of onset $[6,7]$. The in-hospital care and treatment for patients with acute and subacute stroke are very important for stabilizing their medical condition and enabling the recovery of their motor functions [8]. Traditional Chinese medicine (TCM) is a safe treatment $[9,10]$ that has been used for more than 2500 years. Acupuncture and Chinese herbal medicine are the two major forms of TCM therapy [11].

According to the literature, patients who received routine care and TCM had fewer adverse outcomes [12], a lower rate of readmission for circulation-related complications [13], and a reduced risk of mortality [14] during the early stages of stroke recovery. TCM therapy also improves poststroke dysphagia [15], shoulder pain [16], spasticity [17], self-care ability and quality of life [18].

Taiwan's National Health Insurance (NHI) program was established in 1995, and it covers more than $99 \%$ of Taiwan's population [19]. Medicine and TCM are two large categories of healthcare provided by the NHI [20, 21 ], and since NHI programs were started, they covered TCM claims for outpatient clinic services but not for inpatient services. Therefore, the costs of using TCM for stroke inpatients are not covered by the NHI, and hence, these costs were completely covered by the patients before 2006.

The government launched an NHI-sponsored national project in 2006; this project was a pilot scheme of the use of TCM for individuals who had been stroke inpatients for 6 months or less after a confirmed diagnosis of stroke $[12,22]$. The goal of this scheme was to provide better healthcare to inpatients with acute or subacute stroke. Every hospital with TCM physicians providing outpatient services in Taiwan could join this scheme to provide extra TCM as part of routine care for stroke inpatients.

The present study focused on the following questions. What was the trend of using pilot scheme-supported TCM in these inpatients with stroke? If TCM treatment for acute and subacute stroke inpatients was also covered by the NHI, according to the pilot scheme of TCM experiences, what percentage of stroke inpatients were expected to undergo TCM (acupuncture and Chinese herbal medicine) during their first-time stroke hospitalization? Most importantly, what were the potential factors affecting TCM use among inpatients during their hospitalization for first-time stroke? To answer these questions, the researchers conducted a retrospective longitudinal study to examine the characteristics, trends, and important factors affecting NHIsponsored TCM use among first-time stroke inpatients using population-based data from the NHI Research Database (NHIRD). The purpose of this study was to provide clinicians with comprehensive information about TCM use.

\section{Methods}

\section{Source of the data}

In Taiwan, the NHIRD comprises deidentified personal data that are available for research purposes. The database that was used contained longitudinal medical information, including the registry for beneficiaries and contracted medical facilities; details of inpatient and outpatient orders; inpatient and outpatient expenditures; International Classification of Diseases, 9th edition, Clinical Modification (ICD-9-CM) diagnostic codes; and procedure codes of all Taiwanese stroke patients between 1997 and 2013. The researchers collected data on firsttime stroke inpatients from 1997 to 2012. Because these are longitudinal data of all stroke patients from 1997 to 2013, patients who were admitted in 2012 may have been discharged in 2013. However, the researchers still had information about the next years' claims, even for patients who were admitted in late 2012. The researchers did not have the 2014 claim information for discharged first-time stroke inpatients who were admitted in late 2013. Therefore, to avoid bias, the study did not include first-time stroke inpatients who were admitted in 2013. The quality and quantity of NHIRD-related studies have been increasing since the first published study in 2000 [23].

\section{Inclusion/exclusion of study patients}

First, the researchers included patients with inpatient claims, including patient admission data, for stroke diagnosis (ICD-9-CM codes 430-435) from 2006 to 2012. Patients with ICD-9-CM codes 430-435 and 438 (late effects of cerebrovascular disease) for inpatient and outpatient claims, respectively, from 1997 to 2005 were excluded because they were not considered first-time stroke patients. Patients admitted to hospitals outside the TCM scheme were excluded. Patients with no definite date of discharge during hospitalization for first-time stroke were also excluded. Finally, 89,162 first-time stroke patients admitted to hospitals that joined the pilot scheme of TCM were included.

TCM users are defined as stroke inpatients whose claims contain any one of the following codes, especially for the pilot scheme of TCM: supplementary diagnosis 
fees (P33001), Chinese herbal medicine fees (P33021, P33061), acupuncture fees (P33031, P33032), Chinese traumatology treatment fees (P33041), disease management and care fees (P33051-P33053), and supplementary examination fees (P33071-P33074). Among TCM users, patients who used Chinese herbal medicine and acupuncture were defined using their claim data containing codes P33021 and P33061, and codes P33031 and P33032, respectively. Among these 89,162 patients, 7455 were TCM users and 81,707 were TCM nonusers during hospitalization for first-time stroke. The flowchart illustrating patient selection is shown in Fig. 1.

\section{Record selection}

\section{Potential factors affecting TCM use}

Information about the factors that potentially affected TCM use via the pilot scheme was available from the NHIRD and was selected for analysis in the study. Demographic factors, including age $[24,25]$ and sex [24, 25], and clinical factors, such as stroke type [25], length of hospital stay for first-time stroke [22], stroke severity $[22,26]$, stroke comorbidity [24], hospital accreditation level [26], rehabilitation use during hospitalization [22], and the patient's previous experience with TCM use [27], were considered relevant. Socioeconomic factors, such as urbanization [24, 25] and insured amount [24, 25], may also possibly affect medical resource utilization among stroke patients.

Recording patients' information on these factors was based on the following classifications. Age was stratified as $<45$ (young), 45-64, and $\geq 65$ years (old). Sex was recorded as either man or woman. Regarding stroke type, ICD-9-CM codes 433-435 were recorded as ischemia, ICD-9-CM codes 430-432 were recorded as hemorrhage, and both existing ICD-9-CM codes 433435 and 430-432 were recorded as combined ischemia and hemorrhage. The length of hospital stay for firsttime stroke was stratified as 1-7 (within 1 week), 8-14 (1-2 weeks), $15-28$ (2-4 weeks), and $\geq 29$ ( $>4$ weeks) days, and the days of stratification were adopted from a previous NHIRD study on stroke patients [28].

The hospital accreditation level was divided into community hospitals, regional hospitals, and medical centers, which have been used as the 3 hospital accreditation levels in Taiwan [29]. For any given treatment or procedure, higher reimbursements are paid by the Bureau of the NHI to hospitals with a higher accreditation level than to hospitals with a lower accreditation level [30]. However, hospitals with higher accreditation levels are responsible for teaching, training, and research. The accreditation of each hospital is reviewed by the government every 4 years $[29,31,32]$.

Rehabilitation use was defined as the presence of claims for physical, occupational, and speech therapy or any combination thereof. Claims for splint orders were not included in the rehabilitation therapy orders. A patient's previous experience with TCM use was defined as any outpatient claims for TCM in the NHIRD in the past 9 years before the first-time stroke occurrence. For all enrolled patients from 2006 to 2012, the researchers

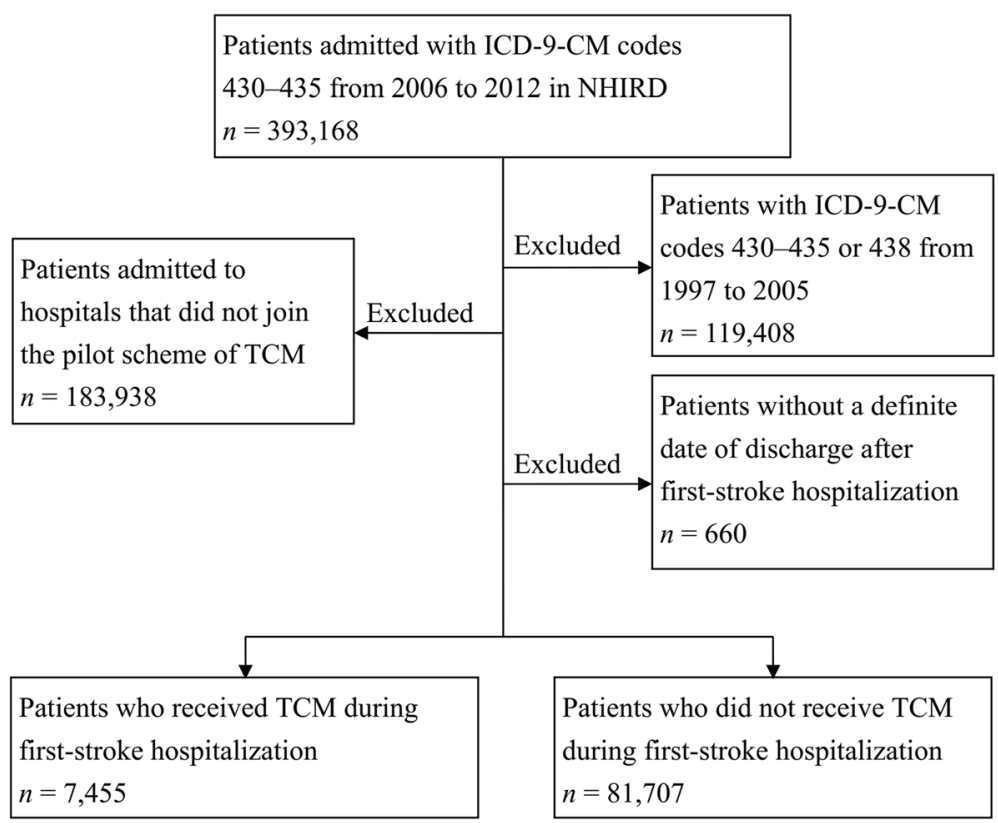

Fig. 1 Flowchart illustrating study patient selection. ICD-9-CM, International Classification of Diseases, 9th edition, Clinical Modification; NHIRD, National Health Insurance Research Database; TCM, traditional Chinese medicine 
aimed to use previous TCM treatment experience before first-time stroke for as many years as possible, resulting in 9 years of claims. This is because data inclusion was based on the year that the program was started (2006), and NHIRD data were only available starting in 1997, which was just 9 years before 2006.

Sung et al. developed a stroke severity index (SSI) score that is highly correlated with the National Institutes of Health Stroke Scale (.743 correlation coefficient) [33] to represent the stroke severity for patients with stroke in the NHIRD. From the analysis of the multiple linear regression model, the summing constant and each individual coefficient of seven predictive features (osmotherapy, urinary catheterization, nasogastric intubation, airway suction, bacterial sensitivity test, general ward stay, and intensive care unit stay) constitute the SSI score. The SSI score ranged between 4.1043 and 27.1125. A higher score indicates greater stroke severity. The 10-point interval, frequently utilized in studies of stroke inpatients [34, 35] for SSI scores of $\geq 20,10-19$, or $<10$ was used to examine stroke severity in these patients.

The Charlson-Deyo comorbidity index (CCI) score was used to estimate the mortality risk from comorbid diseases. The CCI represents a clinical comorbidity index that was calculated from the sum of assigned weights of 17 different diagnostic categories [36, 37]. A higher CCI indicates greater severity of comorbidity. In this study, the researchers used the diagnosis codes of ICD-9-CM from these diagnostic categories (excluding cerebrovascular disease and hemiplegia) to calculate the CCI score. The CCI scores were stratified as $\geq 3,1-2$, or 0 , which have been previously used for translating the severity of comorbidity of stroke inpatients [34, 35].

The patients' residential areas were recorded according to 7 urbanization levels. According to a previous study [38], the 359 townships in Taiwan were categorized into 7 urbanization levels (1, most urbanized; 7, least urbanized) based on population density (people/ $\mathrm{km} 2$ ), the percentage of the population with college or higher educational levels, the percentage of the population aged over 65 years, the percentage of the population that are agricultural workers, and the number of doctors per 100 , 000 people (on the basis of Taiwanese census data from the year 2000).

The NHI set the value of 17,280 New Taiwan dollar (NTD) as the lowest monthly salary (tier 1) and 182,000 NTD as the highest monthly salary (tier 55). In this study, however, the monthly insured amount was categorized as $<576$ USD (lowest monthly salary) $(17,280$ NTD; conversion rate of NTD:USD $=30: 1$ ) and $\geq 576$ USD, considering that most stroke patients were at retirement age and were thus jobless.

\section{Trends of TCM use}

The number of patients who were admitted to hospitals due to first-time stroke and joined the pilot scheme of TCM in each year between 2006 and 2012 was recorded separately. The annual number of patients who received either of the major types of TCM, i.e., Chinese herbal medicine and acupuncture, was also recorded. The annual rate of TCM use by first-time stroke inpatients was obtained as follows: the total number of first-time stroke inpatients utilizing TCM divided by the total number of first-time stroke inpatients in hospitals that joined the pilot scheme of TCM in each year. The annual utilization rates of Chinese herbal medicine and acupuncture among TCM users with first-time stroke were obtained as follows: the total number of first-time stroke inpatients utilizing Chinese herbal medicine divided by the total number of first-time stroke inpatients utilizing TCM in each year and the total number of first-time stroke inpatients utilizing acupuncture divided by the total number of first-time stroke inpatients utilizing TCM in each year.

\section{Statistical methods}

Statistical analyses were performed using SAS Studio version 3.7. To investigate the potential factors affecting TCM use among these stroke inpatients between 2006 and 2012, univariate associations were analyzed for each potential factor using the chi-square test, and Pearson's and Spearman's correlation analyses were used to check for collinearity. TCM was used as a dependent variable and each factor that potentially affected TCM was used as an independent variable in a binary logistic regression. The overall model was used for evaluating the logistic regression model, and the overall model significance for the binary logistic regression was examined using the model coefficients of the Wald chi-square test. The odds ratio (OR), in terms of $\operatorname{Exp}(\beta)$, was calculated for the ratio of the predicted probability of TCM use between two levels of each potential factor. A $P$ value of $<0.05$ was considered statistically significant.

\section{Results}

The prevalence of TCM use among first-time stroke patients who were admitted to hospitals that joined the pilot scheme of TCM increased from 6.5\% in 2006 to $19.6 \%$ in 2012 (Fig. 2). The annual rates of acupuncture use among first-time stroke inpatients ranged from 68.8 to $79.7 \%$, while the rates of Chinese herbal medicine use ranged from 0.8 to $5.1 \%$. The rates of combined acupuncture and Chinese herbal medicine use ranged from 17.8 to $26.1 \%$ (Fig. 3).

Table 1 lists the differences in characteristics between TCM users and TCM nonusers among first-time stroke inpatients between 2006 and 2012. TCM users tended to 


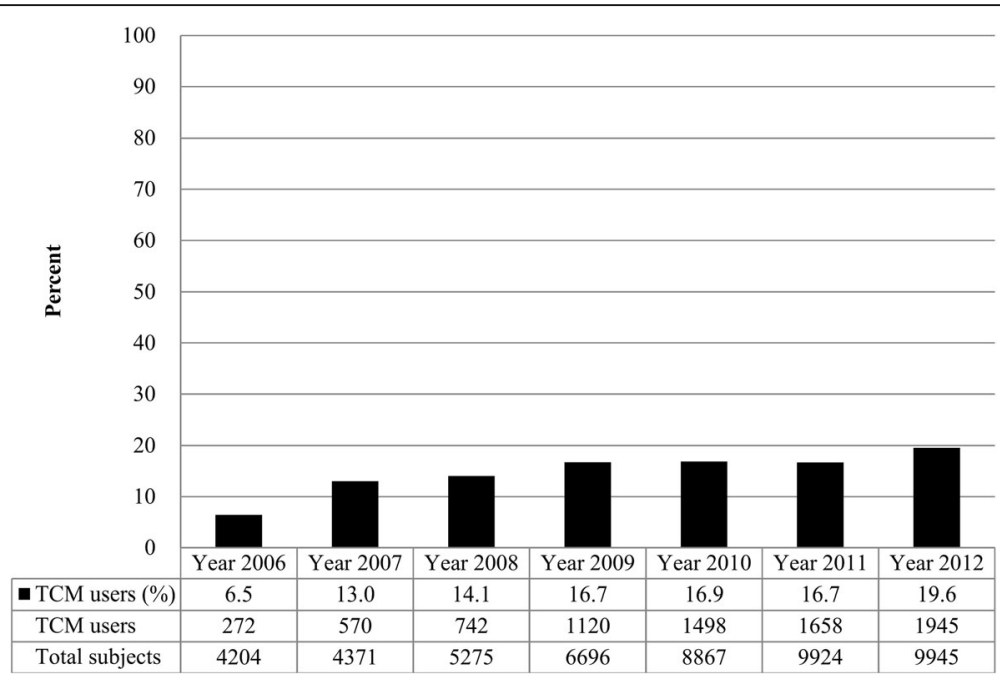

Fig. 2 Rate of TCM use among inpatients with first-time stroke during hospitalization between 2006 and 2012

be younger $(P<0.001)$, have a longer hospital stay $(P<$ $0.001)$, have higher stroke severity $(P<0.001)$, receive rehabilitation during hospitalization $(P<0.001)$, have experience with past TCM use $(P<0.001)$, and have higher insured amounts $(P<0.001)$ than nonusers.

Table 2 shows the results of the logistic regression analysis of factors that predict TCM use among firsttime stroke inpatients. Patients aged < 45 years (OR, 1.58 ; $95 \%$ confidence interval $[\mathrm{CI}], 1.43-1.74)$ and $45-$ 64 years (OR, 1.37; 95\% CI, 1.29-1.45) were more likely to use TCM than patients aged $\geq 65$ years $(P<0.001)$. Men were more likely to use TCM than women (OR,
1.08; 95\% CI, 1.02-1.14; $P=0.007)$. Patients with ischemic stroke (OR, 1.20; 95\% CI, 1.12-1.28; $P<0.001)$ were more likely to use TCM than those with hemorrhagic stroke. Patients hospitalized for first-time stroke for 8 14 days (OR, 2.08; 95\% CI, 1.89-2.29), 15-28 days (OR, 4.10; 95\% CI, 3.72-4.53), and $\geq 29$ days (OR, 11.84; $95 \%$ CI, 10.72-13.08) were more likely to use TCM than those hospitalized for $1-7$ days $(P<0.001)$. Patients with SSI scores of 0-9 (OR, 1.31; 95\% CI, 1.20-1.44) and 1019 (OR, 1.16; 95\% CI, 1.08-1.26) were more likely to use TCM than those with SSI scores of $\geq 20 \quad(P<0.001)$. Patients with CCI scores of 0 (OR, 2.15; 95\% CI, 1.77-

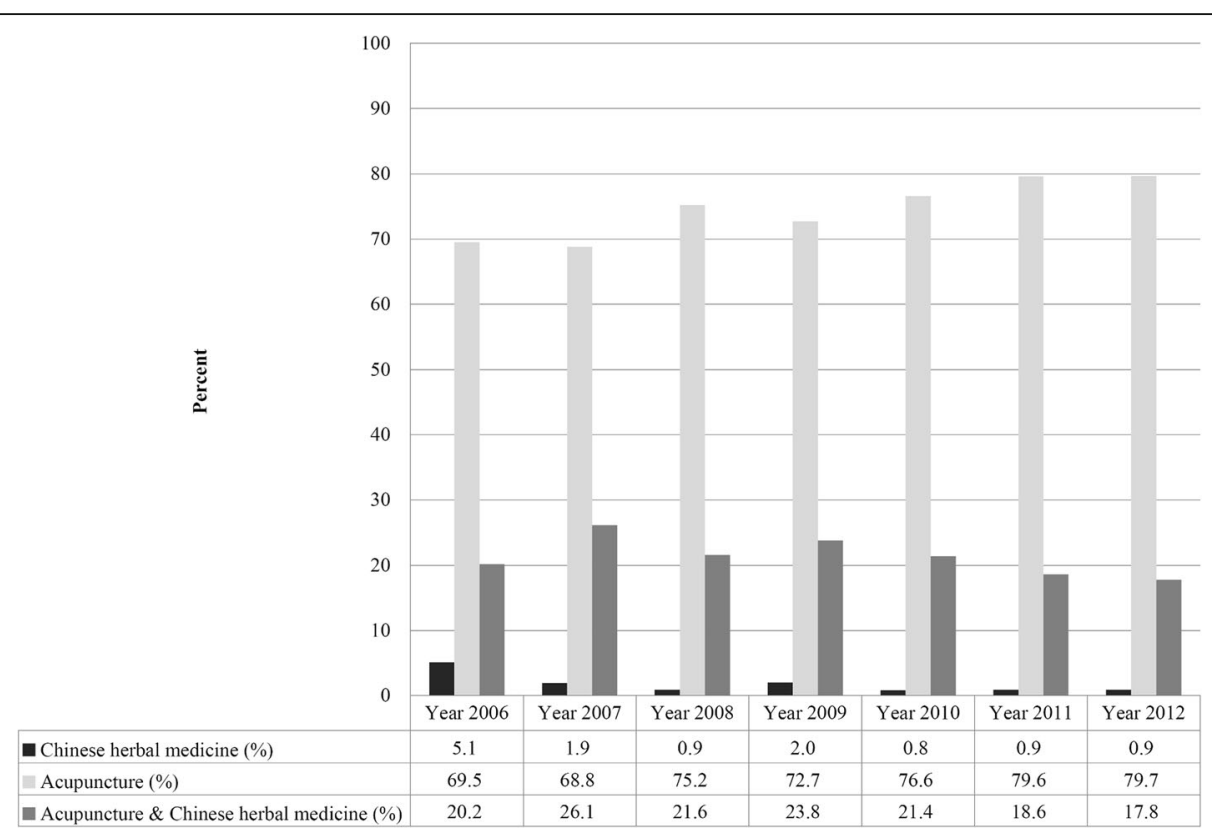

Fig. 3 Rate of Chinese herbal medicine and acupuncture use among inpatients with first-stroke utilizing TCM during hospitalization in 2006-2012 
Table 1 Characteristics of the patients with first-stroke admitted to hospitals participating in the TCM scheme

\begin{tabular}{|c|c|c|c|}
\hline Characteristics & TCM users $(n=7455)$ & TCM non-users $(n=81,707)$ & $P$ \\
\hline \multicolumn{4}{|l|}{ Demographic factors } \\
\hline Age (years) & & & $<0.001$ \\
\hline$\geq 65$ & $3466(46.5 \%)$ & $44,111(54.0 \%)$ & \\
\hline $45-64$ & $3210(43.1 \%)$ & $31,065(38.0 \%)$ & \\
\hline$<45$ & 779 (10.4\%) & $6531(8.0 \%)$ & \\
\hline Sex & & & 0.142 \\
\hline Women & $2912(39.1 \%)$ & $32,627(39.9 \%)$ & \\
\hline Men & $4543(60.9 \%)$ & $49,080(60.1 \%)$ & \\
\hline \multicolumn{4}{|l|}{ Clinical factors } \\
\hline Type of stroke & & & $<0.001$ \\
\hline Hemorrhagic & $2552(34.2 \%)$ & $21,235(26.0 \%)$ & \\
\hline Ischemic & $4752(63.8 \%)$ & $59,433(72.7 \%)$ & \\
\hline Hemorrhagic \& ischemic & $151(2.0 \%)$ & $1039(1.3 \%)$ & \\
\hline Length of hospital stay for first-time stroke (days) & & & $<0.001$ \\
\hline $1-7$ & $783(10.5 \%)$ & $43,133(52.8 \%)$ & \\
\hline $8-14$ & $1244(16.7 \%)$ & $18,442(22.6 \%)$ & \\
\hline $15-28$ & $1645(22.1 \%)$ & $11,211(13.7 \%)$ & \\
\hline$\geq 29$ & $3783(50.7 \%)$ & $8921(10.9 \%)$ & \\
\hline SSI score & & & $<0.001$ \\
\hline$\geq 20$ & $1537(20.6 \%)$ & $9510(11.6 \%)$ & \\
\hline $10-19$ & $2697(36.2 \%)$ & $17,483(21.4 \%)$ & \\
\hline$<10$ & $3221(43.2 \%)$ & $54,714(67.0 \%)$ & \\
\hline $\mathrm{CCl}$ score & & & $<0.001$ \\
\hline$\geq 3$ & $127(1.7 \%)$ & $2321(2.8 \%)$ & \\
\hline $1-2$ & $2938(39.4 \%)$ & $31,313(38.3 \%)$ & \\
\hline 0 & $4390(58.9 \%)$ & $48,073(58.9 \%)$ & \\
\hline Hospital accreditation level & & & $<0.001$ \\
\hline Medical center & $3866(51.9 \%)$ & $44,954(55.0 \%)$ & \\
\hline Regional hospital & $3484(46.7 \%)$ & $36,076(44.2 \%)$ & \\
\hline Community hospital & $105(1.4 \%)$ & $677(0.8 \%)$ & \\
\hline Rehabilitation during hospitalization for first-time stroke & & & $<0.001$ \\
\hline No & $474(6.4 \%)$ & $46,164(56.5 \%)$ & \\
\hline Yes & $6981(93.6 \%)$ & $35,543(43.5 \%)$ & \\
\hline TCM treatment in the past 9 years before first-time stroke & & & $<0.001$ \\
\hline No & $2190(29.4 \%)$ & $26,352(32.2 \%)$ & \\
\hline Yes & $5265(70.6 \%)$ & $55,355(67.8 \%)$ & \\
\hline \multicolumn{4}{|l|}{ Socioeconomic factors } \\
\hline Urbanization residency level & & & $<0.001$ \\
\hline 1 (most urbanized) & $1293(17.4 \%)$ & $15,215(18.6 \%)$ & \\
\hline 2 & $2454(32.9 \%)$ & $25,119(30.7 \%)$ & \\
\hline 3 & $1179(15.8 \%)$ & $13,929(17.1 \%)$ & \\
\hline 4 & $1287(17.3 \%)$ & $13,737(16.8 \%)$ & \\
\hline 5 & $286(3.8 \%)$ & 4007 (4.9\%) & \\
\hline 6 & $471(6.3 \%)$ & $5448(6.7 \%)$ & \\
\hline
\end{tabular}


Table 1 Characteristics of the patients with first-stroke admitted to hospitals participating in the TCM scheme (Continued)

\begin{tabular}{lll}
\hline Characteristics & TCM users $(n=7455)$ & TCM non-users $(n=81,707)$ \\
\hline 7 (least urbanized) & $485(6.5 \%)$ & $4252(5.2 \%)$ \\
Insured amount per month & & \\
$\quad<576$ USD & $3272(43.9 \%)$ & $38,270(46.8 \%)$ \\
$\quad \geq 576$ USD & $4183(56.1 \%)$ & $43,437(53.2 \%)$ \\
\hline
\end{tabular}

Abbreviations: TCM Traditional Chinese medicine; USD United States dollar; SSI Stroke severity index; CCI Charlson-Deyo comorbidity index

2.61) and 1-2 (OR, 2.04; 95\% CI, 1.68-2.48) were more likely to use TCM than those with CCI scores $\geq 3(P<$ $0.001)$. Patients admitted to regional hospitals (OR, 1.41; 95\% CI, 1.34-1.49) and community hospitals (OR, 6.22; 95\% CI, 4.87-7.95) were more likely to use TCM than those admitted to medical centers $(P<0.001)$. Patients receiving rehabilitation during hospitalization for firsttime stroke (OR, 9.16; 95\% CI, 8.28-10.13; $P<0.001$ ) were more likely to use TCM than those without rehabilitation. Patients who received outpatient TCM treatment in the past 9 years before first-time stroke (OR, 1.35; 95\% CI, 1.28-1.43; $P<0.001$ ) were more likely to use TCM than those without outpatient TCM treatment. Patients living in level 2 (OR, 1.18; 95\% CI, 1.091.28), level 4 (OR, 1.21; 95\% CI, 1.10-1.32), and level 7 urbanized areas (OR, 1.38; 95\% CI, 1.22-1.56) were more likely to use TCM than those living in level 1 urbanized areas $(P<0.001)$. Patients with an insured amount of $\geq 576$ USD per month (OR, 1.20; 95\% CI, $1.14-1.27 ; P<0.001)$ were more likely to use TCM than those with an insured amount of $<576$ USD per month.

\section{Discussion}

This study demonstrated that various demographic, clinical, and socioeconomic factors may affect the use of TCM. TCM use among first-time stroke inpatients in Taiwan increased markedly from 2006 to 2012. Among TCM users, approximately $70 \%$ used acupuncture only, fewer than $6 \%$ used Chinese herbal medicine only, and approximately $20-25 \%$ used both acupuncture and Chinese herbal medicine. To the authors' knowledge, this is the first nationwide study to report the prevalence of and factors associated with TCM use among firsttime stroke inpatients in Taiwan.

Complementary and alternative medicine (CAM) is one of the most popular therapies among patients worldwide. In the US, approximately $50 \%$ of stroke survivors use CAM, with acupuncture being the most frequently used therapy [39]. TCM is usually considered a subdivision of CAM. In Korea, 54\% of stroke patients used TCM, and $74 \%$ of them used TCM within 3 months of stroke onset [40]. In this study, the prevalence of TCM use among first-time stroke inpatients increased from $6.5 \%$ in 2006 to $19.6 \%$ in 2012 ; this three-fold increase over 7 years suggests the popularity of TCM compared with other traditional stroke treatments.

In Taiwan, TCM and medicine are two coexisting but independent healthcare systems under the NHI $[20,21]$. However, medical doctors are responsible for treating and caring for stroke inpatients. As mentioned in the introduction, before 2006, there was no TCM scheme $[12,22]$. Stroke inpatients who wished to receive TCM therapy during hospitalization had to pay for it themselves or choose outpatient TCM therapy after discharge. However, outpatient TCM therapy is inconvenient for stroke patients, and they may discontinue treatment due to mobility, transportation, and economic issues. The implementation of the TCM scheme provided an additional and alternative therapy for acute and subacute stroke patients during hospitalization.

The recommendation of first-line physicians or surgeons who provide care for stroke patients may influence the patients' use of TCM [40] because these professionals have the power to decide whether to recommend TCM treatment or not. However, first-line physicians' or surgeons' perceptions of TCM treatment depends on various factors [41], such as their knowledge about the effects of TCM on stroke and hospital policies. The widespread use of TCM is possible, although an increase in TCM use among first-time stroke inpatients was observed in the present study. The next step is to validate the effects of TCM on the recovery of first-time stroke patients. This will enhance physicians' or surgeons' knowledge about the outcomes of therapy, and thus, they may be more receptive to the use of TCM in stroke patients.

In this study, TCM users were more likely to use rehabilitation than TCM nonusers, and the prevalence of TCM use in patients who received rehabilitation was 9.2 times higher than in those who did not. Using rehabilitation during hospitalization for first-time stroke is a strong predictor of TCM use, as shown in a previous study [22]. In Taiwan, stroke rehabilitation therapy is covered by the NHI [42]; therefore, first-time stroke inpatients commonly receive rehabilitation therapy. In Taiwan, approximately $43.6 \%$ of first-time stroke survivors received rehabilitation during their hospitalization for first-time stroke [35]. Clinically, most patients started receiving rehabilitation when their neurological status 
Table 2 Logistic regression analysis of potential factors affecting TCM use among inpatients with first-time stroke

\begin{tabular}{l} 
Characteristics \\
\hline Age (years) \\
$\geq 65$ (reference group) \\
$45-64$ \\
$<45$ \\
Sex \\
Women (reference group) \\
Men \\
Type of stroke \\
Hemorrhagic (reference group) \\
Ischemic \\
Hemorrhagic \& ischemic \\
Length of hospital stay for first-time stroke (days) \\
$1-7$ (reference group) \\
$8-14$ \\
$15-28$ \\
$\geq 29$ \\
SSI score \\
$\geq 20$ (reference group) \\
$10-19$ \\
$<10$ \\
CCI score \\
1-2 (reference group) \\
Regional hospital \\
Community hospital
\end{tabular}

Rehabilitation during hospitalization for first-time stroke

$\begin{array}{lll} & & \\ 1.00 & & \\ 1.37 & 1.29 & 1.45 \\ 1.58 & 1.43 & 1.74\end{array}$

1.00

1.08

1.02

$<0.001$

1.00

1.20

1.12

1.28

1.18

0.97

0.007

1.00

2.08

1.89

2.29

4.10

3.72

4.53

11.84

10.72

13.08

1.00

1.16

1.08

1.26

1.31

1.20

1.44

1.00

2.04

1.68

2.48

2.15

1.77

2.61

1.00

1.41

1.34

1.49

6.22

4.87

7.95

No (reference group)

1.00

Yes

9.16

8.28

10.13

TCM treatment in the past 9 years before first-time stroke

1.00

1.35

Yes

Urbanization residency level

1 (reference group)

1.00

1.18

1.01

1.21

0.92

1.06

1.38

7 (least urbanized)

Insured amount per month

$<576$ USD (reference group)
1.28

1.43

1.09

1.28

0.93

1.11

1.10

1.32

0.80

1.07

0.94

1.20

1.22

1.56
$<0.001$

$<0.001$

$<0.001$ 
Table 2 Logistic regression analysis of potential factors affecting TCM use among inpatients with first-time stroke (Continued)

\begin{tabular}{lllc}
\hline Characteristics & Odds ratio & $95 \%$ Cl Lower & $95 \%$ Cl Upper \\
\hline$\geq 576$ USD & 1.20 & 1.14 & 1.27 \\
\hline Abbreviations: TCM Traditional Chinese medicine; Cl Confidence interval; USD United States dollar; SSI Stroke severity index; CCI Charlson-Deyo comorbidity index
\end{tabular}

and vital signs stabilized [43]. The researchers inferred that the suitable timing of TCM use was similar to that of rehabilitation use in first-time stroke patients. The overlapping period of using TCM and rehabilitation may contribute to positive treatment results.

In this study, TCM use was higher among patients hospitalized for longer periods, especially over 28 days. This result was compatible with that of a previous study [22]. If patients stay in the hospital longer for inpatient rehabilitation, they may obtain more information about TCM and understand it better, thereby increasing their willingness to use TCM. However, a longer hospital stay may depend on different factors, such as the patient's medical condition, the waiting time to transfer to the next medical service to proceed with rehabilitation, and the NHI policy. More studies are needed to analyze these factors in the future.

Patients admitted to regional and community hospitals used TCM more frequently than patients admitted to medical centers. According to this study, among the hospitals joining the TCM scheme from 2006 to 2012, $27.5 \%$ were medical centers, $65 \%$ were regional hospitals, and the remaining $7.5 \%$ were community hospitals. The odds of using TCM were 6.2 times higher in patients admitted to community hospitals than in those admitted to medical centers. However, one article by Chang et al. illustrated that patients admitted to medical centers with a TCM department were more likely to use TCM in Korea [26]. These differences in TCM use between the two studies may be attributed to the different healthcare systems and hospital policies in Korea and Taiwan. In Korea, patients can easily consult TCM doctors at academic medical centers [26]. In Taiwan, although most hospitals have TCM departments, they offer only outpatient services. The researchers considered that most medical centers in Taiwan may have less motivation to encourage their TCM departments to join the TCM scheme because their hospital revenues are always higher than regional and community hospitals. Instead, regional and community hospitals may be more willing to join the TCM scheme because they pursue more profit (i.e., more claims to the NHI).

Liao et al.'s [24] and Weng et al.'s [25] studies showed that TCM use was higher among stroke patients living in urban areas than among those living in rural areas. The present study showed that TCM use was higher among patients living in level 2, 4, and 7 urbanized areas than among those living in level 1 urbanized areas. Although the results did not show the same differences in all levels (compared with level 1), the findings herein still demonstrated that first-time stroke patients living in rural areas are more willing to use TCM. The differences in the findings among our study, Liao et al.'s study [24], and Weng et al.'s study [25] may be because they enrolled patients using outpatient TCM services for 1 year after stroke, whereas we only enrolled patients using TCM during their hospitalization for first-time stroke. In Taiwan, highly urbanized areas have a high density of TCM doctors offering outpatient services [44]. This easy access to outpatient TCM services may influence the healthcare-seeking behaviors of first-time stroke patients after hospital discharge.

Stroke patients have more comorbidities that require professional attention [45]. Physical disability may be due to comorbid diseases [45, 46], which may prevent them from using TCM. Wei et al. found that higher stroke severity and more comorbidities [22] were positively associated with increased TCM use among stroke inpatients. However, our findings showed that lower stroke severity (lower SSI scores) and fewer comorbidities (lower CCI scores) may increase TCM use among first-time stroke inpatients. The difference between our findings and those of Wei et al. may be due to differences in stroke types and hospital types. Wei et al. [22] enrolled 4064 inpatients with ischemic stroke from a medical center in Taiwan, but we enrolled 89,162 inpatients with first-time ischemic and hemorrhagic stroke admitted to hospitals with different accreditation levels in Taiwan. We considered that first-time stroke patients with higher stroke severity and more comorbidities may need intensive care for more days to stabilize their medical condition during hospitalization. Due to the limited days of hospitalization, it is less possible to receive TCM while under intensive care for medical problems related to greater severity and more comorbidities.

The researchers found that TCM use was higher among ischemic stroke inpatients than among those with hemorrhagic stroke. Weng et al. [25] also showed similar trends in outpatient TCM use among patients with different types of stroke. In the literature, acupuncture enabled functional recovery in stroke patients through several mechanisms, such as brain reorganization [47], the regulation of neurochemical release in ischemic stroke patients [48], and the inhibition of TNF- $\alpha / \mathrm{NFK} B$ expression in a rat model of hemorrhagic stroke [49]. Chinese herbal medicine is a popular choice for treating stroke [14]. It is considered to have both in vitro and in vivo angiogenic effects in 
the context of ischemic stroke [50], neuroprotective effects in mice with ischemic stroke via the modulation of molecular targets or genes [51], and improved blood perfusion in the brains of hemorrhagic stroke patients [52]. Although there is more evidence of the benefits of acupuncture or Chinese herbal medicine on the ischemic brain than on the hemorrhagic brain, further studies are needed to prove that TCM is more beneficial in ischemic stroke than in hemorrhagic stroke.

A number of physicians, patients, and their families are concerned about the bleeding tendency and side effects of TCM. However, evidence shows that acupuncture is safe and may only cause minor adverse events, such as pain, dizziness, and fainting [10]. A study applying the Cochrane systematic review methods to 59 kinds of Chinese herbal medicines [9] found that these medicines are relatively free of major adverse effects and are nontoxic to patients with ischemic stroke. However, certain Chinese herbal medicines may increase the risk of bleeding when used in combination with anticoagulants [53]. It must be noted that Chinese herbal medicine may have side effects when used in combination with other medicines [54]. Hence, Chinese herbal medicine, similar to medicine, is relatively safe if used on the basis of therapeutic principles and correct diagnosis. Moreover, adverse pharmacological effects can be avoided if drug compatibility is assessed and suitable drug administration methods are followed [54].

This study had limitations common to retrospective database analyses. First, the diagnoses were sourced from the NHIRD based on ICD-9-CM codes, which may be less accurate than prospective diagnoses. Second, we collected medical claims data that lacked additional information regarding the factors that may influence TCM use. Third, because not all hospitals had TCM services and not all hospitals with TCM services joined the TCM scheme, the prevalence of TCM use may not represent the entire population of first-time stroke patients in Taiwan. Despite these limitations, this study provides a clear profile of the use of pilot scheme-supported TCM among first-time stroke inpatients in Taiwan. Future prospective studies focusing on collecting information on the diagnoses and potential factors that influence TCM use are warranted to address the existing knowledge gap. More studies are needed to provide evidence of better outcomes after TCM use in first-time stroke patients.

\section{Conclusions}

Previous studies have shown that TCM is beneficial for reducing the complication rate, reducing the mortality rate and enhancing the quality of life of stroke patients. The TCM pilot scheme is a quick and convenient way to provide TCM to willing first-time stroke patients. The overall trend showed positive growth and greater acceptance of TCM use among first-time stroke patients. This study clearly demonstrated that the important predictors for TCM use among first-time stroke inpatients in Taiwan were age, sex, area of residence, income status, length of hospital stay, hospital accreditation level, type and severity of stroke, presence of comorbidities, receiving rehabilitation, and previous experience with outpatient TCM services. These findings could provide information on TCM use among first-time stroke inpatients to first-line medical staff and hospital policy makers.

\section{Abbreviations}

TCM: Traditional Chinese medicine; NHI: National health insurance; NHIR D: National health insurance research database; ICD-9-CM: International classification of diseases, 9th edition, clinical modification; SSI: Stroke severity index; CCl: Charlson-Deyo comorbidity index; CAM: Complementary and alternative medicine

\section{Acknowledgements}

The authors thank the Health Information and Epidemiology Laboratory of the Chang Gung Memorial Hospital, Chiayi Branch, for the comments and assistance in data analysis.

\section{Authors' contributions}

WSC, HCH, YFC, and CMC conceptualized the study. YWC, ML, and CMC performed the statistical analysis. WSC, KYL, and CMC contributed to the interpretation of the data. WSC wrote the manuscript. All the authors have read and approved the final manuscript.

\section{Funding}

This research did not receive any specific grant from funding agencies in the public, commercial, or not-for-profit sectors.

\section{Availability of data and materials}

The datasets used and/or analyzed during the current study are available from the corresponding author on reasonable request.

\section{Ethics approval and consent to participate}

The current study protocol was approved by the Institutional Review Board for Human Studies of the Chang Gung Memorial Hospital (approval no. 201800032B1), which waived the need for patient informed consent because the study utilized secondary de-identified information.

Consent for publication

Not applicable.

\section{Competing interests}

The authors declare that they have no competing interests.

\section{Author details}

${ }^{1}$ Department of Physical Medicine and Rehabilitation, Chang Gung Memorial Hospital, Chiayi, No.6, W. Sec., Jiapu Rd.,, Puzih City, Chiayi County 613, Taiwan. ${ }^{2}$ Department of Physical Medicine and Rehabilitation, Jing Mei Hospital, Taipei, Taiwan. ${ }^{3}$ School of Medicine, College of Medicine, Chang Gung University, Taoyuan, Taiwan. ${ }^{4}$ Department of Nursing, Chang Gung University of Science and Technology, Chiayi Campus, Chiayi, Taiwan. ${ }^{5}$ Department of Natural Biotechnology, Nanhua University, Dalin, Chiayi, Taiwan. ${ }^{6}$ Center for Musculoskeletal Regenerative Medicine, Chang Gung Memorial Hospital, Chiayi, Taiwan. ${ }^{7}$ Department of Physical Medicine and Rehabilitation, Xiamen Chang Gung Hospital, Xiamen, China. ${ }^{8}$ Jinan Rehabilitation Clinic, Tainan, Taiwan. ${ }^{9}$ Department of Neurology, Chang Gung Memorial Hospital, Chiayi, Taiwan. ${ }^{10}$ School of Traditional Chinese Medicine, College of Medicine, Tzu Chi University, Hualien, Taiwan. 
Received: 24 August 2018 Accepted: 26 July 2020

Published online: 06 August 2020

\section{References}

1. Bonita R, Stewart A, Beaglehole R. International trends in stroke mortality: 1970-1985. Stroke. 1990;21(7):989-92.

2. O'Mahony PG, Thomson RG, Dobson R, Rodgers H, James OFW. The prevalence of stroke and associated disability. J Public Health. 1999;21(2): 166-71.

3. Hu HH, Sheng WY, Chu FL, Lan CF, Chiang BN. Incidence of stroke in Taiwan. Stroke. 1992;23(9):1237-41.

4. Lin HC, Lin YJ, Liu TC, Chen CS, Chiu WT. Urbanization and stroke prevalence in Taiwan: analysis of a nationwide survey. J Urban Health. 2007; 84(4):604-14.

5. Hsieh Fl, Chiou HY. Stroke: morbidity, risk factors, and care in Taiwan. J Stroke. 2014;16(2):59-64

6. Kelly-Hayes M, Wolf PA, Kase CS, Gresham GE, Kannel WB, D'Agostino RB. Time course of functional recovery after stroke: the Framingham study. Neurorehabil Neural Repair. 1989;3(2):65-70.

7. Jorgensen HS, Nakayama H, Raaschou HO, Vive-Larsen J, Stoier M, Olsen TS. Outcome and time course of recovery in stroke. Part II: time course of recovery. The Copenhagen stroke study. Arch Phys Med Rehabil. 1995;76(5): 406-12.

8. Langhorne P, Pollock A. What are the components of effective stroke unit care? Age Ageing. 2002;31(5):365-71.

9. Wu B, Liu M, Liu H, Li W, Tan S, Zhang S, et al. Meta-analysis of traditional Chinese patent medicine for ischemic stroke. Stroke. 2007;38(6):1973-9.

10. Xu M, Li D, Zhang S. Acupuncture for acute stroke. Cochrane Database Syst Rev. 2018;3:CD003317

11. Zhou J, Qu F. Treating gynaecological disorders with traditional Chinese medicine: a review. Afr J Tradit Complement Altern Med. 2009;6(4):494-517.

12. Chang CC, Chen TL, Chiu HE, Hu CJ, Yeh CC, Tsai CC, et al. Outcomes after stroke in patients receiving adjuvant therapy with traditional Chinese medicine: a nationwide matched interventional cohort study. J Ethnopharmacol. 2016;177:46-52.

13. Chiu HE, Hung YC, Chang KC, Shih CC, Hung JW, Liu CW, et al. Favorable circulatory system outcomes as adjuvant traditional Chinese medicine (TCM) treatment for cerebrovascular diseases in Taiwan. PLoS One. 2014;9(1): e86351.

14. Chang CC, Lee YC, Lin CC, Chang CH, Chiu CD, Chou LW, et al. Characteristics of traditional Chinese medicine usage in patients with stroke in Taiwan: a nationwide population-based study. J Ethnopharmacol. 2016; 186:311-21.

15. Zhang JH, Wang D, Liu M. Overview of systematic reviews and metaanalyses of acupuncture for stroke. Neuroepidemiology. 2014;42(1):50-8.

16. Lee JA, Park SW, Hwang PW, Lim SM, Kook S, Choi Kl, et al. Acupuncture for shoulder pain after stroke: a systematic review. J Altern Complement Med. 2012;18(9):818-23.

17. Lim SM, Yoo J, Lee E, Kim HJ, Shin S, Han G, et al. Acupuncture for spasticity after stroke: a systematic review and meta-analysis of randomized controlled trials. Evid Based Complement Alternat Med. 2015;2015:870398.

18. Shen PF, Kong L, Ni LW, Guo HL, Yang S, Zhang LL, et al. Acupuncture intervention in ischemic stroke: a randomized controlled prospective study. Am J Chin Med. 2012:40(4):685-93.

19. Wu TY, Majeed A, Kuo KN. An overview of the healthcare system in Taiwan. London J Prim Care. 2010;3(2):115-9.

20. Yeh ML, Chiu WL, Wang YJ, Lo C. An investigation of the use of traditional Chinese medicine and complementary and alternative medicine in stroke patients. Holist Nurs Pract. 2017;31(6):400-7.

21. Chang LC, Huang N, Chou YJ, Lee CH, Kao FY, Huang YT. Utilization patterns of Chinese medicine and Western medicine under the National Health Insurance Program in Taiwan, a population-based study from 1997 to 2003. BMC Health Serv Res. 2008;8:170.

22. Wei YC, Sun MF, Chang KC, Chang CJ, Hung YC, Lin YJ, et al. Pilot scheme of health policy in stroke adjuvant acupuncture therapy for acute and subacute ischemic stroke in Taiwan. Evid Based Complement Alternat Med. 2011;2011:689813.

23. Chen YC, Yeh HY, Wu JC, Haschler I, Chen TJ, Wetter T. Taiwan's National Health Insurance Research Database: administrative health care database as study object in bibliometrics. Scientometrics. 2010;86(2):365-80.
24. Liao CC, Lin JG, Tsai CC, Lane HL, Su TC, Wang HH, et al. An investigation of the use of traditional chinese medicine in stroke patients in Taiwan. Evid Based Complement Alternat Med. 2012;2012:387164.

25. Weng SW, Chen TL, Yeh CC, Liao CC, Lane HL, Lin JG, et al. An investigation of the use of acupuncture in stroke patients in Taiwan: a national cohort study. BMC Complement Altern Med. 2016;16(1):321.

26. Chang H, Kwon YD, Yoon SS. Use of acupuncture therapy as a supplement to conventional medical treatments for acute ischaemic stroke patients in an academic medical Centre in Korea. Complement Ther Med. 2011;19(5): 256-63.

27. Yam W, Wilkinson JM. Is acupuncture an acceptable option in stroke rehabilitation? A survey of stroke patients. Complement Ther Med. 2010; 18(3-4):143-9.

28. Chen $\mathrm{CM}$, Yang $\mathrm{YH}$, Lee $\mathrm{M}$, Chen $\mathrm{KH}$, Huang SS. Economic evaluation of transferring first-stroke survivors to rehabilitation wards: a 10-year longitudinal, population-based study. Top Stroke Rehabil. 2020;27(1):8-14.

29. Huang P. An overview of hospital accreditation in Taiwan, Republic of China. Int J Health Plann Manage. 1995;10(3):183-91.

30. Lin CM, Li CY. Assessment of medical resource utilization for Taiwanese children hospitalized for intracranial injuries. Injury. 2014;45(4):690-5.

31. Wung $\mathrm{CH}$. The reform of the hospital accreditation system in Taiwan. World Hosp Health Serv. 2008;44(1):14-5 18.

32. Joint Commission of Taiwan. https://www.jct.org.tw/cp-1127-1106-22fc4-2. html. Accessed 28 Mar 2020.

33. Sung SF, Hsieh CY, Kao Yang YH, Lin HJ, Chen CH, Chen YW, et al. Developing a stroke severity index based on administrative data was feasible using data mining techniques. J Clin Epidemiol. 2015;68(11):1292-300.

34. Chen CM, Lee M, Yang YH, Huang SS, Lin CH. Association between clinical and laboratory markers and 5-year mortality among patients with stroke. Sci Rep. 2019;9(1):11521.

35. Chen $\mathrm{CM}$, Yang $\mathrm{YH}$, Chang $\mathrm{CH}$, Chen PC. Effects of transferring to the rehabilitation Ward on long-term mortality rate of first-time stroke survivors: a population-based study. Arch Phys Med Rehabil. 2017;98(12):2399-407.

36. Charlson ME, Pompei P, Ales KL, MacKenzie CR. A new method of classifying prognostic comorbidity in longitudinal studies: development and validation. J Chronic Dis. 1987:40(5):373-83.

37. Deyo RA, Cherkin DC, Ciol MA. Adapting a clinical comorbidity index for use with ICD-9-CM administrative databases. J Clin Epidemiol. 1992:45(6):613-9.

38. Liu CY, Hung YT, Chuang YL. Incorporating development stratification of Taiwan townships into sampling design of large scale health interview survey. J Health Manag. 2006;4:1-22.

39. Shah SH, Engelhardt R, Ovbiagele B. Patterns of complementary and alternative medicine use among United States stroke survivors. J Neurol Sci. 2008;271(1-2):180-5

40. Shin Yl, Yang CY, Joo MC, Lee SG, Kim JH, Lee MS. Patterns of using complementary and alternative medicine by stroke patients at two university hospitals in Korea. Evid Based Complement Alternat Med. 2008; 5(2):231-5.

41. Chung VC, Hillier S, Lau CH, Wong SY, Yeoh EK, Griffiths SM. Referral to and attitude towards traditional Chinese medicine amongst western medical doctors in postcolonial Hong Kong. Soc Sci Med. 2011;72(2):247-55.

42. Lee HC, Chang KC, Huang YC, Lan CF, Chen JJ, Wei SH. Inpatient rehabilitation utilization for acute stroke under a universal health insurance system. Am J Manag Care. 2010;16(3):e67-74.

43. Lai CL, Tsai MM, Luo JY, Liao WC, Hsu PS, Chen HY. Post-acute care for stroke - a retrospective cohort study in Taiwan. Patient Prefer Adherence. 2017;11:1309-15.

44. Shih CC, Su YC, Liao CC, Lin JG. Patterns of medical pluralism among adults: results from the 2001 National Health Interview Survey in Taiwan. BMC Health Serv Res. 2010;10:191.

45. Janus-Laszuk B, Mirowska-Guzel D, Sarzynska-Dlugosz I, Czlonkowska A. Effect of medical complications on the after-stroke rehabilitation outcome. NeuroRehabilitation. 2017;40(2):223-32.

46. Roth EJ, Mueller K, Green D. Stroke rehabilitation outcome: impact of coronary artery disease. Stroke. 1988;19(1):42-7.

47. Shen Y, Li M, Wei R, Lou M. Effect of acupuncture therapy for postponing Wallerian degeneration of cerebral infarction as shown by diffusion tensor imaging. J Altern Complement Med. 2012;18(12):1154-60.

48. Zhang H, Kang T, Li L, Zhang J. Electroacupuncture reduces hemiplegia following acute middle cerebral artery infarction with alteration of serum NSE, S-100B and endothelin. Curr Neurovasc Res. 2013;10(3):216-21. 
49. Liu H, Sun X, Zou W, Leng M, Zhang B, Kang X, et al. Scalp acupuncture attenuates neurological deficits in a rat model of hemorrhagic stroke. Complement Ther Med. 2017;32:85-90.

50. Seto SW, Chang D, Jenkins A, Bensoussan A, Kiat H. Angiogenesis in ischemic stroke and Angiogenic effects of Chinese herbal medicine. J Clin Med. 2016;5(6):56

51. Wang HW, Liou KT, Wang YH, Lu CK, Lin YL, Lee IJ, et al. Deciphering the neuroprotective mechanisms of Bu-yang Huan-wu decoction by an integrative neurofunctional and genomic approach in ischemic stroke mice. J Ethnopharmacol. 2011;138(1):22-33.

52. Liang Q, Li X, Zhang H. Effects of nao yi-an granule on hemorheological indexes and RCD (erythrocyte deformability) in patients with hemorrhagic stroke. Bull Hunan Med Univ. 1998:23(6):545-8.

53. Tsai HH, Lin HW, Lu YH, Chen YL, Mahady GB. A review of potential harmful interactions between anticoagulant/antiplatelet agents and Chinese herbal medicines. PLoS One. 2013;8(5):e64255.

54. He J, Kwon Y, Li C, Zhang XQ, Zhao JG. Several considerations in using traditional Chinese patent medicine for cerebral infarction. Chin J Integr Med. 2012;18(8):571-4.

\section{Publisher's Note}

Springer Nature remains neutral with regard to jurisdictional claims in published maps and institutional affiliations.

Ready to submit your research? Choose BMC and benefit from:

- fast, convenient online submission

- thorough peer review by experienced researchers in your field

- rapid publication on acceptance

- support for research data, including large and complex data types

- gold Open Access which fosters wider collaboration and increased citations

- maximum visibility for your research: over $100 \mathrm{M}$ website views per year

At BMC, research is always in progress.

Learn more biomedcentral.com/submissions 